\title{
Hommage à François Fourrier
}

\section{In Memoriam François Fournier}

\author{
P. Charbonneau - C. Chopin - F. Saulnier \\ (C) SRLF et Lavoisier SAS 2019
}

\section{Le collègue, leader de la discipline (Professeur P. Charbonneau)}

Je n'ai connu François que dans les années 1980 lorsque le DESC interrégional de réanimation a été créé et initié dans la région nord-ouest par Claude Chopin. Il me revient en mémoire notre première réunion pédagogique à la Ferme Saint-Siméon sur les hauteurs d'Honfleur. L'enseignant lumineux m'est apparu ainsi que son humanisme, sa grande sensibilité que ses deux ouvrages mettront en lumière, lui qui savait rester humble et discret.

Ainsi pendant plus de 20 ans, nous avons eu la chance de découvrir le médecin, le merveilleux enseignant, l'homme qui apporta tant, par ses travaux, dans le suivi des malades atteints du syndrome de Guillain-Barré ou dans la difficile compréhension des troubles complexes de la coagulation lors des états sévères.

$\mathrm{Au}$ fil des années, enseignants et étudiants s'enrichissaient à son contact.

La SRLF dont il a été président lui doit aussi beaucoup, car sa probité intellectuelle et sa démarche scientifique éclairaient la Société, mais aussi parce qu'il a su y faire entrer de plain-pied toutes celles et tous ceux qui travaillent et donnent le meilleur d'eux-mêmes pour le soin des patients de réanimation. Ainsi, infirmières et kinésithérapeutes eurent leur place au Congrès annuel de la Société.

François Fourrier a consacré sa vie professionnelle à la réanimation à travers toutes les instances représentatives où

\footnotetext{
P. Charbonneau

Ancien président de la Société de réanimation de langue française,

Caen, France

C. Chopin

Professeur honoraire, ancien chef de service de réanimation polyvalente, université de Lille-II, Lille, France

F. Saulnier ( $\square)$

Ancienne présidente du Collège des enseignants en médecine intensive et réanimation, Lille, France e-mail : fabienne.saulnier@chru-lille.fr
}

il a su donner à la discipline la place qu'elle mérite dans le domaine de la santé comme dans le domaine universitaire.

Pour tout cela et pour tout ce que d'autres ont pu exprimer au cours de l'hommage qui lui fut rendu il y a peu, Merci, un grand Merci.

\section{L'enseignant et le pédagogue, respectueux et respecté (Professeur F. Saulnier)}

François s'est toujours engagé avec conviction, enthousiasme et rigueur dans tous les aspects de la fonction de PUPH, et son investissement en tant qu'enseignant a été exceptionnel et exemplaire.

Outre ses qualités intrinsèques de pédagogue et son talent d'orateur qui lui permettaient d'accrocher l'auditoire lors de ses interventions en cours ou conférences, il avait une créativité singulière et était toujours à l'affût de méthodes et d'organisations pédagogiques nouvelles. Comme on met à jour une bibliographie, il renouvelait régulièrement les modalités pédagogiques des enseignements qu'il assurait et surtout les programmes dont il avait la responsabilité, adaptant l'ensemble à l'évolution des attentes des enseignés et aux changements docimologiques en utilisant largement mais avec discrimination les progrès des techniques de communication, notamment l'enseignement à distance.

François avait une vision de l'enseignement qui ne se limitait pas à la transmission du savoir scientifique, mais aussi de l'humanisme qui l'animait et du comportement avec les patients et les familles. Ses « tours » au lit du patient étaient attendus par tous ceux qui travaillaient avec lui. Les plus jeunes ont certes été marqués par ses capacités de synthèse « à la volée » de phénomènes compliqués, comme la cascade de la coagulation par exemple, qui, en l'écoutant, devenaient simples et limpides. Mais ce dont ils se souviennent aussi, et peut-être surtout, ce sont ses remarques : « il faut frapper avant d'entrer dans la chambre »; "vous $n$ 'avez pas dit bonjour en entrant » ; ...

Cet aspect de la prise en charge en réanimation, qui lui tenait à cœur et qu'il voulait transmettre, s'est concrétisé 
avec le concept de « Mieux vivre en réanimation » qu'il a développé, centré sur les patients et les familles, et pour lequel il a dirigé une conférence de consensus dont les conclusions font toujours référence.

Il a assuré des missions de responsabilité tant au niveau local qu'interrégional et national. Chacun connaît les nombreuses fonctions qu'il a occupées depuis sa nomination en 1986. Il serait inutile et vain de chercher l'exhaustivité : membre du conseil de pédagogie et du conseil de la FMC, créateur et responsable d'un enseignement à choix libre $(E C L)$ « réponses à l'agression » pour les étudiants du deuxième cycle, initiateur et responsable du département universitaire de réanimation et urgences médicales (DURUM), créateur et directeur de deux diplômes universitaires, l'un pour les médecins (diplôme universitaire de réanimation polyvalente : DURP) et l'autre pour les infirmiers (ières) et kinésithérapeutes (diplôme universitaire soins en réanimation [DUSER]) à la faculté de médecine de Lille ; coordonnateur interrégional du DESC pour le Nord-Ouest ; membre de notre sous-section 48-02 du Conseil national des universités $(\mathrm{CNU})$ et président du Collège des enseignants de réanimation (CNER, actuel CeMIR).

La création du DURP et ensuite du DUSER, qui ont d'emblée rencontré un vif succès, attirant des étudiants de toutes les régions de France et dépassant ainsi très largement la faculté de médecine de Lille, a correspondu à sa volonté de former des professionnels de réanimation de qualité et s'est inscrite dans sa vision exigeante de la mission des soignants.

Il a été un des premiers coordonnateurs interrégionaux nord-ouest du DESC perpétuant tout à la fois niveau scientifique et manifestations conviviales. Ses remarques et commentaires souvent percutants au cours des séances d'enseignement suscitaient une certaine appréhension parmi les étudiants, et même parfois parmi les autres enseignants, mais l'intérêt et le respect prenaient toujours le dessus, car ils étaient pertinents et instructifs, empreints de bienveillance, d'une expérience et d'une expertise incontestables au service des étudiants. De plus, tous savaient que son exigence envers les autres n'avait d'égale que son intransigeance envers luimême.

C'est sous son mandat de président du Collège que la réflexion sur la création d'un DES spécifique à notre spécialité a réellement débuté. Convaincu par l'importance des enjeux de cette transformation pédagogique et structurelle pour l'avenir de notre discipline, il pressentait cependant, avec hauteur de vue, les difficultés qui ne manqueraient pas de survenir. Il en a posé les premières briques, notamment en élaborant un programme d'enseignement des bases de la réanimation, fédérant pour cela l'ensemble des enseignants de la discipline. C'est en grande partie à partir de ces documents que l'enseignement par audioconférences en e- learning pour les DES MIR phase socle, tel que disponible aujourd'hui sur SIDES et le site du Collège, a vu le jour.

Aujourd'hui, le DURP et le DUSER perdurent avec un succès et une audience qui ne faiblissent pas. Le département universitaire de réanimation et urgences médicales (DURUM) est devenu le DUMIR et représente une structure incontournable pour les instances locales qui nous dirigent, tant sur le plan facultaire qu'hospitalier. Enfin, le DES de médecine intensive-réanimation (MIR) a vu le jour en 2017.

Ces quelques exemples témoignent, s'il en était nécessaire, de l'acuité de sa vision prospective de l'avenir et de sa force de conviction. Celles et ceux qui ont eu pour tâche de continuer à faire vivre ses initiatives ne peuvent que le remercier pour ce qu'il a impulsé et lui rendre hommage.

Tout cela et plus, c'était François l'enseignant et le pédagogue.

\section{L'élève et l'ami (Professeur C. Chopin)}

Salut François, mon élève, mon ami, mon frère. Pour moi tu es toujours là. As-tu entendu tes éloges ? Tellement dithyrambiques surtout celles des petits. Je ne peux pas en rajouter, tu finirais de ne plus me croire. C'est de ta faute après tout. Dans la logique des choses, l'élève favori se doit de faire l'éloge de son patron. Toi tu as inversé le cérémonial et cela te plaît. Je te vois rire de mon embarras. Je sais que tu aimes secouer l'ordre établi. Mais en plus de 40 ans de vie commune, d'écoute et de respect mutuel, nous n'avons traversé que quelques orages et le plus souvent alors que je tentais de calmer ton ardeur à vouloir étrangler un administratif à la comptabilité trop prétentieuse...

Quel excellent médecin et brillant enseignant tu étais ! Que l'élève ait pu dépasser le maître, ce qui était redouté de la plupart des patrons faisait au contraire ma fierté. Ensemble nous avons éveillé chez les plus jeunes l'importance de comprendre la physiopathologie des signes et des symptômes, et de la démarche qui mène au diagnostic.

$\mathrm{Tu}$ étais aussi un très bon pianiste. Un soir de congrès alors que, solitaires, nous déambulions dans les salons d'un grand hôtel parisien, nous parvînmes à l'oreille les notes du concerto en $m i$ mineur de Chopin, évidemment... Comme aimanté par la mélodie, tu t'installas au plus près du pianiste qui, bientôt, dut te laisser le tabouret. Tu jouas ainsi 45 minutes pour notre plaisir et celui des clients de l'hôtel.

Bien que connaissant les qualités littéraires de François, qualités qui transparaissaient à travers la moindre de ses écritures, y compris professionnelles, parfois trop pour l'objectif et le récipiendaire, je fus bluffé par ses deux livres publiés, excusez du peu, chez Albin Michel. Sans doute, François aurait écrit et publié encore beaucoup de livres. Les lignes suivantes attestent de son talent. Elles sont prémonitoires : 
«Il faut écouter les voix qui s'éteignent, les souffles ténus qui expirent. Des organismes qui disparaissent, des énergies qui se dissipent, des esprits ou des âmes qui rejoignent des divins inconnus. Des humains qui survivent et d'autres qui meurent ».
François Fourrier, Le Souffle, la Conscience et la Vie, pages 1-5, 2017 Albin Michel, Ed.

Salut l'artiste ! 